\title{
Philosophiques
}

\section{Notice critique : Reconstructing Reason and Representation - Murray Clarke}

\section{Michael Bishop}

Volume 34, numéro 2, automne 2007

URI : https://id.erudit.org/iderudit/017425ar

DOI : https://doi.org/10.7202/017425ar

Aller au sommaire du numéro

Éditeur(s)

Société de philosophie du Québec

ISSN

0316-2923 (imprimé)

1492-1391 (numérique)

Découvrir la revue

Citer ce document

Bishop, M. (2007). Notice critique : Reconstructing Reason and RepresentationMurray Clarke. Philosophiques, 34(2), 367-374. https://doi.org/10.7202/017425ar d'utilisation que vous pouvez consulter en ligne.

https://apropos.erudit.org/fr/usagers/politique-dutilisation/ 


\title{
Notice critique: Reconstructing Reason and Representation - Murray Clarke
}

\author{
MICHAEL BISHOP \\ Université de la Floride
}

Nul ne peut raisonnablement nier que nos esprits sont le produit de l'évolution. Le problème va au-delà de cette généralisation. La psychologie évolutionniste $(\mathrm{PE})$ gère le problème en proposant une histoire très spécifique de la façon dont nos esprits ont été formés par l'évolution: quelque part au cours du Pléistocène, un évènement génétique s'est produit et a occasionné, chez certains de nos prédécesseurs chasseurs-cueilleurs, un module mental (un processeur computationnel) qui a efficacement contribué à relever les défis quant à leur capacité à survivre et se reproduire. Conséquemment, le module acquis par les chasseurs-cueilleurs leur a donné un avantage reproductif sur leurs contemporains qui en étaient privés, et donc, après plusieurs générations, il est devenu prévalent parmi nos ancêtres. Ce processus s'est répété de nombreuses fois pour produire chez nos ancêtres beaucoup (peut-être des milliers) de modules innés et spécialisés (Barkow, Cosmides \& Tooby 1992). La sélection naturelle nous a légué des esprits massivement modulaires.

Depuis environ une décennie, de sérieuses inquiétudes sur les fondements biologiques de la PE ont été soulevées (voir Buller, 2005). Le livre ambitieux et intéressant de Murray Clarke, Reconstructing Reason and Representation, ne soulève pas ces soucis à leur propos. Il vise plutôt à extraire de la PE un ensemble complet de perspectives sur la connaissance et l'esprit humain. Ce compte rendu mettra l'accent sur trois des thèmes principaux de Clarke: sa défense de la modularité massive, sa solution partielle au problème de la disjonction (ou erreur de représentation), et sa proposition selon laquelle la connaissance est fragmentée.

\section{La modularité massive}

Le cas standard de "l'effet lié au contenu » de la modularité massive commence avec la découverte empirique selon laquelle la valeur du raisonnement des agents cognitifs dépend du contenu de ses conditionnelles (Cosmides, 1989). Considérez ces deux règles conditionnelles (Clarke, 2004, 92):

a. Si une personne a la note " $\mathrm{D}$ ", alors son document doit être identifié code « 3 ».

b. Si une personne boit de la bière, alors elle doit avoir plus de 20 ans.

En dépit du fait qu'elles partagent la même structure logique, les gens trouvent plus facile de raisonner correctement à propos de $b$ que de $a$. Qu'est-ce qui explique cet effet lié au contenu? Une hypothèse veut que les humains aient 
un «module de détection des tricheurs ", lequel est activé à chaque fois qu'un sujet identifie une situation sociale d'échange (Gigerenzer et Hug, 1992). Dans l'exemple ci-dessus, $b$ déclenche notre module de détection des tricheurs (donc, nous savons qu'il faut vérifier l'âge du consommateur de bière et la boisson du mineur) mais a ne le fait pas (Clarke, 2004, 7-12, 90-98). L'objection la plus sérieuse à cet argument pose une hypothèse rivale: l'effet lié au contenu peut être parfaitement bien expliqué par des mécanismes généraux opérant sur des connaissances innées. En conséquence, la découverte de l'effet lié au contenu ne nous donne pas de bonnes raisons d'accepter que l'esprit soit massivement modulaire. Je crois que cette objection a été proposée explicitement pour la première fois par Richard Samuels (1998), bien que, comme celui-ci le note, elle soit implicite dans le travail de Carey et Spelke (1994). (Pour d'autres versions de l'argument, voir Fodor, 2000, et Buller, 2005.)

Clarke défend que le problème de cette objection est qu'elle présuppose une version invraisemblablement forte de la modularité massive - nommément, que l'esprit est entièrement modulaire. Mais il insiste sur l'idée que la modularité massive est une hypothèse inclusive. Il maintient que l'esprit est "dominé » par les modules, mais il reconnaît la possibilité que certaines pensées d'ordre supérieur peuvent être le résultat d'un processeur général agissant sur des ensembles spécifiques ou généraux de connaissance (2004, 7, 36, 28-29, 138-140). Étant donné cette perspective sur la situation dialectique, Clarke souligne que l'hypothèse proposée par Samuels (comme Fodor et les autres) n'est pas incompatible avec l'hypothèse de la modularité massive. Elle laisse donc la modularité massive indemne. Le problème est que dans sa réponse, Clarke se représente mal l'objection. Quand Samuels suggère que l'effet lié au contenu peut être expliqué par une hypothèse rivale, il conclut explicitement que les partisans de la PE ont failli «à nous fournir de bonnes raisons de préférer [la modularité massive] à une image rivale de l'esprit ", et il en résulte que l'hypothèse de la modularité massive "est à la fois sans garantie et immotivée " (Samuels, 1998, 575, soulignement ajouté). Le point est que les partisans défendant la PE sur la base de données psychologiques, comme Clarke, ne nous donnent apparemment aucune raison de croire que l'esprit est dominé par des modules spécialisés plutôt que par des processus généraux agissant sur des connaissances spécifiques. Ces données peuvent être parfaitement expliquées sans qu'on fasse appel à aucun module.

À certaines occasions, Clarke saisit la portée réelle de l'objection. Par exemple, lorsqu'il caractérise la version de Fodor comme suit : «En effet, [Fodor] affirme qu'il est possible d'employer un mécanisme d'apprentissage général non modulaire à côté de toutes sortes d'informations innées » $(2004,28)$. Clarke esquive cette objection, mais seulement en évoquant l'hypothèse de la modularité massive. En répondant à Fodor, il fait endosser aux partisans de la modularité massive l'affirmation qu'il est impossible pour l'esprit d'être seulement constitué d'un "mécanisme général d'apprentissage non modulaire couplé uniquement avec de l'information perceptuelle» (2004, 29, soulignement 
ajouté). Mais cette conclusion n'est pas équivalente à la version canonique de Clarke concernant l'hypothèse de la modularité massive (qui, je le rappelle, soutient que l'esprit est dominé par des modules spécialisés mais ne s'épuise pas dans ceux-ci). Pour nous en rendre compte, prenons un nativiste comme Fodor. Il sera certainement d'accord avec le fait que la cognition supérieure n'implique pas de mécanismes généraux d'apprentissage agissant seulement sur de l'information perceptuelle. Mais nous pouvons néanmoins nier, en restant cohérent, que l'esprit est dominé par des processeurs spécifiques.

Un défi sérieux pour les partisans de la PE est d'expliquer pourquoi il est plausible de croire que l'esprit est dominé par des modules spécialisés plutôt que par des processeurs généraux agissant sur des connaissances spécifiques. D'après ce que j'ai pu constater, ce n'est pas un défi que Clarke a relevé avec succès.

\section{Le problème de la disjonction (ou des erreurs de représentation)}

Supposez qu'un exemplaire d'un type d'état « $\mathrm{R}$ » apparaisse presque toujours en présence de chevaux mais apparaisse plutôt occasionnellement en présence d'autres mammifères quadripèdes. Que représente « R» ? Intuitivement, il semble évident qu'il représente les chevaux. Auquel cas les exemplaires de " R », en présence d'autres quadripèdes, constituent une erreur de représentation. Mais peut-être « $\mathrm{R}$ » représente-t-il des chevaux ou des mules? Auquel cas les exemplaires de « $\mathrm{R}$ » sont des erreurs de représentation uniquement quand la chose représentée est autre chose qu'un cheval ou une mule. Ou peut-être « R » représente-t-il tous les objets, et seulement ceux qui tendent à susciter les exemplaires de « $\mathrm{R}$ »? Auquel cas les exemplaires de " $\mathrm{R}$ » ne peuvent pas être des erreurs de représentation. Le problème de la disjonction (ou de l'erreur de représentation) est donc ceci : en vertu de quel fait les exemplaires de « $\mathrm{R}$ » occasionnent-ils des erreurs de représentation?

La solution partielle de Clarke au problème de la disjonction fait appel à la différence entre le domaine propre d'un module (l'environnement dans lequel l'évolution a sélectionné ce module) et le domaine actuel de ce module (l'environnement dans lequel le module est actif actuellement). La «théorie du fossé » de Clarke explique les erreurs de représentation comme le résultat de la présence d'un fossé entre le domaine propre et le domaine actuel du module. Supposez que les castors possèdent un module cognitif qui tende à signaler « $\mathrm{X}$ » quand un prédateur est à proximité ; ce signal amène un castor en train de nager à claquer sa queue sur la surface de l'eau. Ce module est sélectionné par l'évolution parce qu'il signale les prédateurs, et cela, pour Clarke, est ce que le type d'état mental «X» représente. Mais prenez les castors vivant au zoo de San Diego. Puisqu'il n'y a plus de prédateurs dans leur environnement, tout exemplaire de l'état mental "X $\mathrm{X}$ est une erreur de représentation. Toutefois, cette erreur de représentation est le résultat du mode " propre » de fonctionnement du module dans un environnement différent de celui pour lequel le module a été adapté. "Les erreurs de représentations ne concernent pas l'esprit »(Clarke, 2004, 60). Elles adviennent parce que l'environnement 
dans lequel l'évolution a sélectionné le module associé avec l'état mental est différent de l'environnement dans lequel le module agit actuellement. (Clarke reconnaît que cette solution est partielle, puisque "dans la mesure où cette partie de l'esprit est susceptible d'être non modulaire, une analyse différente serait requise pour rendre compte de ces cas » [2004, 58]).

La théorie du fossé de Clarke soulève une inquiétude manifeste: un état mental est-il une erreur de représentation lorsque son module cognitif associé est dans son domaine propre (quand il n'y a pas de fossé entre le domaine propre et le domaine actuel) ? Prenez un environnement où l'évolution a sélectionné le module chez les prédécesseurs des castors qui les a menés à claquer leur queue. Pendant les (présumées) milliers d'années durant lesquelles le module a été sélectionné, il s'est sûrement déclenché en présence d'un animal qui n'était pas un prédateur, disons un ongulé végétarien. Ce cas est-il un exemple d'erreur de représentation? Il semble que Clarke soit forcé à le nier puisqu'il n'y a pas de fossé entre le domaine propre et le domaine actuel. Mais Clarke rejette l'implication: "Bien sûr, je ne voulais pas suggérer qu'aucune erreur n'advient dans le [domaine propre] ; le Pléistocène n'était pas parfait» (2004, 60). Mais si l'erreur de représentation est le résultat d'un module qui n'est pas dans son domaine propre, comment les erreurs de représentations peuvent-elles advenir quand ce module est dans son domaine propre? Voilà ce qu'en dit Clarke:

Il n'y a aucun domaine dont le domaine propre n'est pas contextuel... Donc, par exemple, la période du Pléistocène était un domaine propre relativement à nos domaines actuels (contemporains). Mais, la période du Pléistocène était aussi un domaine actuel relativement au domaine propre du Miocène. Penser autrement est supposer que la sélection naturelle n'est apparue pour la première fois que durant la période du Pléistocène $(2004,65)$.

Dans ce passage, Clarke suggère que les erreurs de représentation adviennent parce que le domaine propre du Pléistocène possède un domaine propre antérieur dans le Miocène. Mais cela est confus. Les domaines propres sont définis relativement à des modules particuliers : le domaine propre d'un module est l'environnement dans lequel l'évolution a sélectionné ce module. Si le module a été sélectionné durant une certaine période du Pléistocène, alors, par définition, c'était son domaine propre. Il est incompréhensible de se prononcer sur le «domaine propre » pour «nos domaines actuels (contemporains)» ou pour "la période du Pléistocène ». Bien entendu, la théorie du fossé peut expliquer comment les erreurs de représentation advenaient durant le Pléistocène - si l'esprit de nos ancêtres du Pléistocène consistait en modules dont le domaine propre précédait le Pléistocène. Mais cela ne résout pas l'énigme de la façon dont la théorie du fossé explique les erreurs de représentation quand le module associé avec l'état mental qui constitue une erreur de représentation est dans son domaine propre. 


\section{Modules fiables et connaissance}

Clarke défend que l'évolution a sélectionné des modules cognitifs hautement fiables. Bien qu'il admette que nos mécanismes judicatifs ne sont pas toujours parfaitement fiables $(2004,78)$, il ne dit pas jusqu'à quel point la sélection naturelle a été efficace dans le façonnage des mécanismes judicatifs fiables. Tous nos modules cognitifs sont-ils hautement fiables - au moins dans leur domaine propre ? Ou seulement la plupart d'entre eux? Ce n'est pas clair, mais Clarke donne le ton de sa discussion au début du chapitre quatre: "Il y a peutêtre [...] des cas où "l'ignorance est source de bonheur" ou "peu de connaissance est une chose dangereuse", mais généralement les ensembles de fausses croyances ne peuvent tout simplement pas, le plus souvent, être un avantage pour les humains. En fait, je suis presque embarrassé de devoir défendre l'affirmation que les fausses croyances diminuent l'adaptabilité de l'organisme [inclusive fitness], donc sa négation me semble entièrement absurde» $(2004,75)$.

À qui Clarke attribue-t-il cette affirmation «absurde » ? Il n'est pas du tout évident que Stephen Stich, sa principale cible dans ce chapitre, l'affirme (voir 1990, 121). Ce que défend Stich, c'est que l'évolution a pu sélectionner des mécanismes inférentiels faillibles qui commettent beaucoup d'erreurs mineures peu coûteuses en échange de ne (presque) jamais faire d'erreurs majeures très coûteuses. Il décrit un scénario dans lequel un organisme emploie une stratégie d'évitement des erreurs majeures pour identifier les aliments toxiques. L'organisme en vient parfois à considérer erronément qu'un aliment est toxique mais (presque) jamais faussement qu'un aliment n'est pas toxique.

La stratégie d'évitement des risques, qui est faillible et prompte à l'erreur, a bien pu être favorisée par la sélection naturelle. Parce que la sélection naturelle est indifférente à la vérité; elle s'occupe seulement du succès reproductif. Et du point de vue du succès reproductif, il est souvent préférable d'être sauf (ou trompé) que désolé (Stich, 1990, 62).

L'argument de Stich montre qu'il est possible pour l'évolution de sélectionner des mécanismes faillibles de formation des croyances, mais Clarke défend «que les arguments conceptuels à propos de la simple possibilité physique font peu de travail empirique » $(2004,77)$. L'ironie, c'est que pendant que Clarke rejette l'argument de Stich parce qu'il met l'accent uniquement sur "ce qui "pourrait bien" être le cas " $(2004,77)$, il procède immédiatement à la défense du fait que les croyances vraies sont instrumentalement bénéfiques, « les processus fiables auraient bien pu être sélectionnés » (2004, 78, soulignement ajouté). Équité oblige, Clarke présente aussi deux autres arguments. Le premier repose sur l'hypothèse que "l'on pourrait avoir autant de raison de croire que le raisonnement fiable a été sélectionné pour ce propos que de croire que des capacités visuelles faibles ont été sélectionnées pour celui-ci » (2004, 90). Le second argument fait appel au fait que nous raisonnons de façon assez fiable dans les cas de détection des tricheurs, et de la même manière lorsque les problèmes sont formulés en termes de fréquences plutôt que de probabilités 
(2004, 90-98, 118-119). Comme Clarke le reconnaît, aucun de ces arguments ne nous donne de raisons fortes de croire que tous les milliers de modules cognitifs que l'évolution est censée avoir sélectionné sont fiables — ou étaient fiables dans leurs domaines propres $(2004,99)$.

Sommes-nous dans une impasse opposant deux arguments aussi plausibles ? Peut-être que non. Il existe des appuis empiriques qui représentent un sérieux défi à l'optimisme évolutionnaire de Clarke concernant nos habiletés cognitives. Shelley Taylor et ses collègues ont affirmé que «l'optimisme irréaliste à propos de l'avenir permet généralement une meilleure adaptabilité en ce qu'il promeut [...] des sentiments d'amour propre, la capacité à et envers les autres, la persistance et la créativité dans la poursuite d'objectifs, et la capacité à gérer efficacement le stress » (Taylor et al., 1992, 460). Les recherches suggèrent que les optimistes minimalement irréalistes s'en tirent mieux quant aux problèmes de santé que les réalistes, ils tendent à guérir plus rapidement que les réalistes et ils ont des taux de rémission plus élevés que les réalistes (Scheier et al., 1989; Fitzgerald et al., 1993). Alors que l'optimisme irréaliste semble avoir des effets salutaires sur la santé et la survie, la plupart d'entre nous semblent posséder une surprenante et persistante capacité à adopter et protéger de l'infirmation les croyances trop optimistes nous concernant (Taylor, 1989, chapitre 4).

Nous sommes enclins à croire que nous possédons une foule de caractéristiques socialement désirables, et que nous sommes exempts de la plupart de celles qui sont socialement indésirables. Par exemple, une large majorité de la population générale pense qu'elle est plus intelligente, plus encline à l'équité, moins préjudiciable et plus habile derrière un volant d'automobile que la personne moyenne. Ce phénomène est si récurrent et omniprésent qu'il est désormais connu sous le nom de "l'effet Lac Wobegon", à la suite de la communauté fictive de Garrison Keillor où « les femmes sont fortes, les hommes beaux et tous les enfants sont supérieurs à la moyenne ». Une enquête établie sur un million d'élèves sortants du secondaire montre que $70 \%$ d'entre eux pensent qu'ils se situent au-dessus de la moyenne quant à leur habileté de meneur et seulement $2 \%$ pensent qu'ils sont en dessous de la moyenne. En termes de socialisation avec les pairs, tous les étudiants pensent qu'ils sont au-dessus de la moyenne, $60 \%$ pensent qu'ils sont parmi les premiers $10 \%$ et $25 \%$ pensent se trouver dans le premier $1 \%$ ! De peur que d'aucuns croient qu'une autoévaluation si enflée n'a cours que dans les esprits des étudiants inexpérimentés du secondaire, il faudrait souligner qu'une enquête sur les professeurs d'université montre que 94 \% d'entre eux se pensent plus compétents dans leur travail que la moyenne de leurs collègues (Gilovich, 1991, 77).

Si la PE est sur la bonne voie, il n'est pas «absurde» de supposer que l'évolution a pu favoriser au moins quelques modules cognitifs du type Lac Wobegon.

Clarke défend une conception largement fiabiliste de la connaissance (où celle-ci est une croyance produite qui est vraie et fiable). Les représentations vraies qui sont "abritées" dans les modules darwiniens innés et fiables sont 
des connaissances innées (2004, 140-141); et les représentations vraies qui sont les outputs de modules darwiniens fiables sont des connaissances empiriques (2004, 141-142). Clarke identifie un avantage important de coupler le fiabilisme avec la psychologie évolutionnaire : la promesse d'une solution graduelle au problème de la généralité, au problème de trouver une solution principielle à l'identification des processus mentaux dont la fiabilité fixe le statut justificatif des croyances empiriques. Une croyance empirique est une connaissance si elle est une croyance vraie produite par un module darwinien fiable et inné $(2004,64)$. Jusque-là, tout va bien.

Le problème, de mon point de vue, vient de ce que Clarke défend une conception fragmentée de la connaissance. Sa défense s'effectue en deux parties. Premièrement, il offre les raisons suivantes de croire que la connaissance est un genre naturel $(2004,133-136)$ : la connaissance existe; ce n'est pas un "genre conceptuel " parce que si c'en était un, elle serait relative culturellement (ce qu'elle n'est pas). Alors, en conséquence, elle doit être un genre naturel. Clarke soutient l'affirmation que la connaissance doit être un genre ou n'être rien du tout sur la base que si d'aucuns revendiquent qu'elle est autre chose, "[il] commence à perdre prise sur ce qui constitue l'objet de la discussion. Appelez cela la conception " "mystérieuse" de la connaissance» $(2004,135)$. Mais, assurément, cet argument est trop permissif. Supposez que nous nommions la chose dans mon bureau « la chose du bureau de Mike ». Nous pouvons employer le cadre des arguments de Clarke pour montrer que, parce que la chose du bureau de Mike existe et n'est pas culturellement relative, ce doit être un genre naturel. Mais c'est entièrement faux. Clarke ne reconnaît pas la possibilité des salmigondis. Peut-être ce que nous nommons «connaissance » est-il un assemblage hétéroclite d'états qui ne peuvent pas jouer un rôle prééminent dans les lois causales. En fait, Clarke se trouve sur le point d'embrasser cette perspective dans la seconde partie de sa défense d'une conception fragmentée de la connaissance. Il affirme qu' « il n'existe pas de genre naturel unique qui se trouve être la connaissance » $(2004,145)$. Cela parce que chaque module cognitif possède sa propre histoire causale, et que les genres naturels sont définis (en partie) en termes de lois causales responsables de leur unité. Mais au lieu de conclure que la connaissance est un salmigondis fragmenté, il affirme que la connaissance est un « ensemble de genres naturels » fragmentés (2004, 128, 146).

La conception de la connaissance fragmentée de Clarke est intrigante mais sommaire. En fait, l'acquisition de la connaissance pour la PE impliquerait la coopération de multiples modules différents. Par exemple, supposez que j’aie appris pour la première fois que $p$ par témoignage. Cela implique la mémoire à court terme, plusieurs processeurs linguistiques et des processeurs d'information de nature sociale (par exemple, le locuteur est-il digne de confiance? est-ce qu'il s'exprime littéralement?). Ensuite, mes connaissances ont été enrichies par l'expérience (ce qui implique aussi la coopération de plusieurs modules différents), et c'est à présent par la mémoire à long terme que tout 


\section{4 · Philosophiques / Automne 2007}

cela est maintenu. À chaque fois que mes connaissances sont étayées par l'opération d'un autre module, il semblerait, dans la perspective de Clarke, que mes connaissances deviennent une instanciation d'un nouveau genre naturel. Si cela est acceptable et s'il existe des milliers de modules, alors il pourrait facilement y avoir des millions de combinaisons différentes de modules qui ont pris part à l'établissement et au maintien des différentes instanciations des connaissances. La connaissance, selon la perspective de Clarke, semble être fractionnée en millions de fragments. Mais quel intérêt théorique ou explicatif peut nous rapporter une telle sur-taxonomisation ? J. D. Trout et moi-même (2005) avons argumenté que l'épistémologie est obsédée, à tort, par la catégorie de la connaissance. Je suggère que la perspective de Clarke donne raison à notre dérogation face à la connaissance, puisqu'elle mène à une conception de celle-ci trop éclatée pour être théoriquement utile.

\section{Bibliographie}

Barkow, J., L. Cosmides et J. Tooby. The Adapted Mind: Evolutionary Psychology and the Generation of Culture, Oxford, Oxford University Press, 1992.

Bishop, M. et J. D. Trout, Epistemology and the Psychology of Human Judgment, New York, Oxford University Press, 2005.

Buller, D. Adapting Minds: Evolutionary Psychology and the Persistent Quest for Human Nature, Cambridge, MA, The MIT Press, 2005.

Carey, S. et E. Spelke. Domains-Specific Knowledge and Conceptual Change, in Mapping the Mind: Domain-specificity in Cognition and Culture, L. Hirshfield et S. Gelman (dir.), Cambridge, Cambridge University Press, 1994.

Clarke, M. Reconstructing Reason and Representation, Cambridge, MA, The MIT Press, 2004.

Cosmides, L. The Logic of Social Exchange: Has Natural Selection Shaped How Humans Reason, Ethology and Sociobiology, 10, 51-97, 1989.

Fitzgerald, T., H Tennen, G. Affleck, G. Pransky. The relative importance of Dispositional Optimism and Control Appraisals in Quality of Life after Coronary Bypass surgery, in Journal of Behavioral Medicine, 1993, 16, 25-43.

Fodor, J. The Mind Doesn't Work That Way, Cambridge, MA, The MIT Press, 2000.

Gigerenzer, G. et K. Hug. Domain-specific Reasoning: Social Contracts, Cheating and Perspective Change, Cognition, 1992, 43, 127-171.

Gilovich, T. How We Know What Isn't So, New York, The Free Press, 1991.

Samuels, R. Evolutionary Psychology and the Massive Modularity Hypothesis, British Journal for the Philosophy of Science, 1998, 49, 575-602.

Scheier, M., K. Matthews, J. Owens, G. Magovern, R. Lefebvre, R. Abbott et C. Carver. Dispositional optimism and Recovery from Coronary Artery Bypass Surgery: The Beneficial Effects on Physical and Psychological Well-Being, in Journal of Personality and Social Psychology, 1989, 57, 1024-1040.

Stich, S. The Fragmentation of Reason, Cambridge, MIT Press, 1990.

Taylor, S. Positive Illusions: Creative Self-Deception and the Healthy Mind, New York, Basic Books, 1989. 